\title{
AIAA-2001-ABSTRACT On the Development of a Gridless Inflation Code for Parachute Simulations
}

J. H. Strickland, G. F. Homicz, A. A. Gossler, W. P. Wolfe, and V. L. Porter

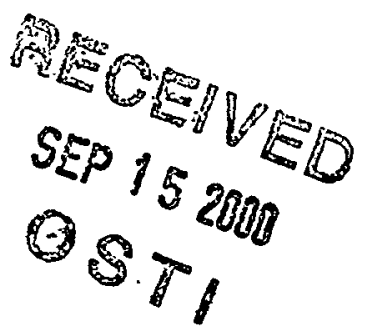

Engineering Sciences Center Sandia National Laboratories Albuquerque, NM 87185-0835

NOTE:

ABSTRACT SUBMITTED: SEPTEMBER 1, 2000

\section{6th AIAA Aerodynamic Decelerator Systems Technology Conference}

May 21-24, 2001/ Boston, MA 
(this page intentionally left blank) 


\section{DISCLAIMER}

This report was prepared as an account of work sponsored by an agency of the United States Government. Neither the United States Government nor any agency thereof, nor any of their employees, make any warranty, express or implied, or assumes any legal liability or responsibility for the accuracy, completeness, or usefulness of any information, apparatus, product, or process disclosed, or represents that its use would not infringe privately owned rights. Reference herein to any specific commercial product, process, or service by trade name, trademark, manufacturer, or otherwise does not necessarily constitute or imply its endorsement, recommendation, or favoring by the United States Government or any agency thereof. The views and opinions of authors expressed herein do not necessarily state or reflect those of the United States Government or any agency thereof. 


\section{DISCLAIMER}

Portions of this document may be illegible in electronic image products. Images are produced from the best available original document. 


\title{
On the Development of a Gridless Inflation Code for Parachute Simulations ${ }^{\dagger}$
}

\author{
J. H. Strickland ${ }^{1}$, G. F. Homicz ${ }^{2}$, \\ A. A. Gossler ${ }^{3}$, W. P. Wolfe ${ }^{4}$, and \\ V. L. Porter \\ Engineering Sciences Center \\ Sandia National Laboratories \\ Albuquerque, NM 87185-0835
}

\begin{abstract}
In this paper we present the current status of an unsteady 3D parachute simulation code which is being developed at Sandia National Laboratories under the Department of Energy's Accelerated Strategic Computing Initiative (ASCI). The Vortex Inflation PARachute code (VIPAR) which embodies this effort will eventually be able to perform complete numerical simulations of ribbon parachute deployment, inflation, and steady descent. At the present time we have a working serial version of the uncoupled fluids code which can simulate unsteady 3D incompressible flows around bluff bodies made up of triangular membrane elements. A parallel version of the code has just been completed which will allow us to compute flows over complex geometries utilizing several thousand processors on one of the new DOE "teraFLOP" computers.
\end{abstract}

\section{INTRODUCTION}

Motivations for Sandia's development of the VIPAR code have been given previously by Peterson ${ }^{1}$ et al. as well as Behr ${ }^{2}$ et al. Aside from the obvious enhancement of Sandia's parachute

\footnotetext{
T This work performed at Sandia National Laboratories supported by the U. S. Dept. of Energy under contract No. DE-AC04-94AL85000.

1 Distinguished Member Technical Staff, Associate Fellow AIAA

2 Senior Member Technical Staff, Member AIAA

3 Post Doc

4 Principal Member Technical Staff, Associate Fellow AIAA

5 Senior Member Technical Staff
}

design capability, the primary motivation is the need to know whether or not the present stockpile life of the war reserve parachutes associated with nuclear weapons can be. safely extended. Furthermore, the ASCI program was established by DOE to provide weapon system engineers and designers with reliable computational predictive tools for stewardship of the nuclear weapons stockpile.

About two years ago we began work on the unsteady 3D parachute simulation code which is based upon a gridless vortex method. We chose this method because it does not require bodyfitted grids in the fluid volume for the deforming structure. Additionally, it only requires one to track the evolution of the wake vorticity field which is itself spacially localized. The VIPAR code architecture is similar to that developed by Strickland ${ }^{3}$ for the axisymmetric parachute code VPARA which was later successfully coupled by Sundberg ${ }^{4}$ to the structural dynamics code PRONTO2D.

The present code has not been coupled to any structural code as of this writing but will easily accept transient geometry specification from any source including an appropriate structural dynamics code. The present code contains a motion generator which can be used to produce a large class of rigid body motions. In the upcoming fiscal year (FY2001) we plan to couple 
the parallelized version of VIPAR to the structural dynamics code PRESTO which is a next generation ASCI tool to replace PRONTO.

\section{OVERVIEW OF METHOD}

\section{Fluid Mechanics}

The initial surface geometry is generated from triangular shell elements using a meshing tool such as PATRAN and is written into a data base file for subsequent input into VIPAR. An example of such a mesh is shown in Figure 1 for a simple four-ring parachute geometry which we have labeled the "MAVEN" geometry. Without going into any details, MAVEN is simply an experimental validation program within ASCI.

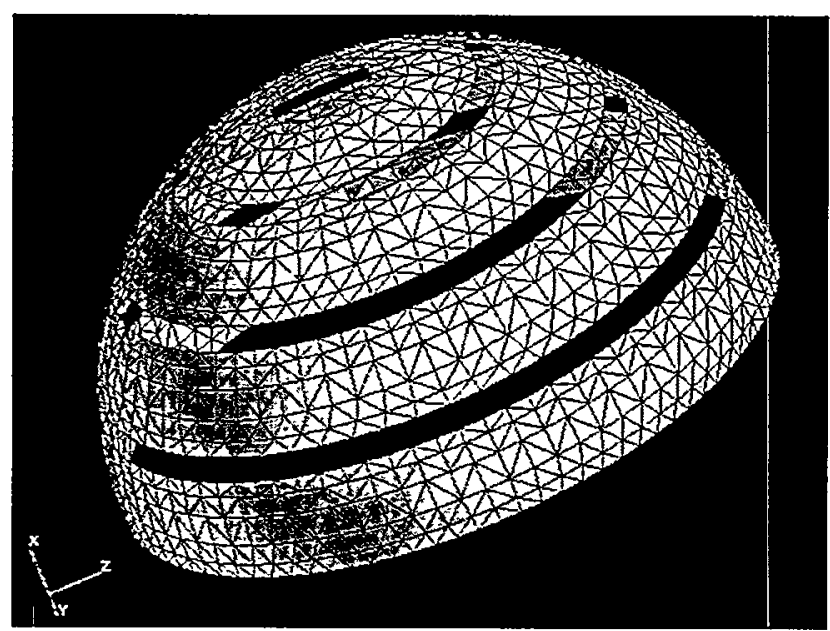

Figure 1. Example Fluid Surface Mesh

The computation at the beginning of each time step requires that the normal velocity boundary condition be satisfied. In order to satisfy this boundary condition, a vortex sheet is placed on the parachute surface which produces a normal velocity at the surface. The strengths are adjusted so that the sum of the normal velocity perturbations from the freestream, the wake vorticity, and the new surface vorticity equal the normal velocity of the surface itself. This requires that a set of linear equations be solved for the unknown surface vorticity sheet strengths. The linear equation set is based upon boundary element theory. In the present version of VIPAR one can choose between a smoothed low order boundary element method which assumes piecewise constant velocity potential jumps on each triangular element or a higher order boundary element method developed by Gharakhani ${ }^{5,6}$ which assumes piecewise linear potential jumps on each triangular element.

Next, the surface vorticity sheet is split into two sheets (one on each side of the surface) so as to satisfy the tangential velocity boundary condition (no slip). In this operation, the sum of the two split sheet vorticity strengths is equal to the original sheet strength so that the normal boundary condition is still satisfied. The sheets are then moved (diffused) away from the surface and converted to vortons with Gaussian cores.

Once the vorticity has been shed from the surface, it is convected, stretched, and diffused in the flow. In the present version of VIPAR there is no merging or splitting of vortex elements. The diffusion model used in VIPAR contains a stretching term which enhances diffusion. For low Reynolds number flows, diffusion may cause the vorton cores to overlap excessively. In future versions of VIPAR we plan to implement merging and splitting algorithms to better manage vortex element strain and growth due to stretching and diffusion, respectively, as well as to control the total number of vortons in the flow. We also plan to implement a particle redistribution algorithm which has been developed by Gharakhani ${ }^{7}$ for diffusion and may perhaps be extended to include the vortex stretching term.

\section{Parallel Computing}

The parallelization of the VIPAR code has been a top priority for the last two years. Homicz ${ }^{8,2}$ parallelized the first VIPAR code module which was the fast multipole solver that computes the velocity field from the vorticity field. This code module has a major impact on VIPAR CPU time. Very good parallel efficiencies have been demonstrated: better than $80 \%$ with at least 1,000 vortex elements held by each processor, and in excess of $90 \%$ with 10,000 or more vortices/ processor. The algorithm also exhibits excellent 
scalability, having been run for model problems with up to $8,000,000$ vortex elements, using as many as 2,048 processors.

The first serial version of VIPAR containing modules which represent all of the basic fluid physics was finished at the end of FY1999. Parallelization of this version has just been completed and CPU performance data will soon be available as the code is exercised.

The parallel efficiency is expected to be good since considerable care has been taken to carefully balance computing and memory needs equally across the processors while minimizing communication costs.

\section{Fluid-Structure Coupling}

The coupling of the fluids code VIPAR with the structural dynamics code PRESTO will be the top priority during FY2001. This coupling will occur in the context of the SIERRA software development environment (Taylor ${ }^{9}$ et al.). This environment consists of an object-oriented framework of software services that are common to Sandia's suite of mechanics application codes and a set of software tools for managing the development process.

The structural dynamics code PRESTO is the SIERRA version of PRONTO3D which was originally developed by Taylor and Flanagan ${ }^{10}$. Modifications will have to be made in PRESTO in order to account for the behavior of textile material and to interface smoothly with the VIPAR code. At the present time VIPAR is not fully integrated into the SIERRA environment, although this effort is well underway.

Since the parachute mass is relatively small as compared to the mass of fluid which is accelerated during deployment, VIPAR and PRESTO must be tightly coupled. It is not sufficient to simply have VIPAR pass surface pressures to PRESTO and PRESTO pass surface shape and velocity back to VIPAR. This is exacerbated by the fact that PRESTO is an explicit code and many structural time steps are required for each fluid time step. Thus VIPAR will pass "added fluid mass" information to
PRESTO which will then be utilized in the equations of motion resident within PRESTO.

\section{PRELIMINARY RESULTS}

\section{Impulsively Started Disk}

We are presently in the process of exercising VIPAR in order to compare its 3D simulations with the experimental and simulated unsteady axisymmetric flows over disks presented by Higuchi ${ }^{11}$ et al. In Figure 2, we show the wake development in two different planes through the center line of the disk as predicted by VIPAR after 2.5 radii of travel.

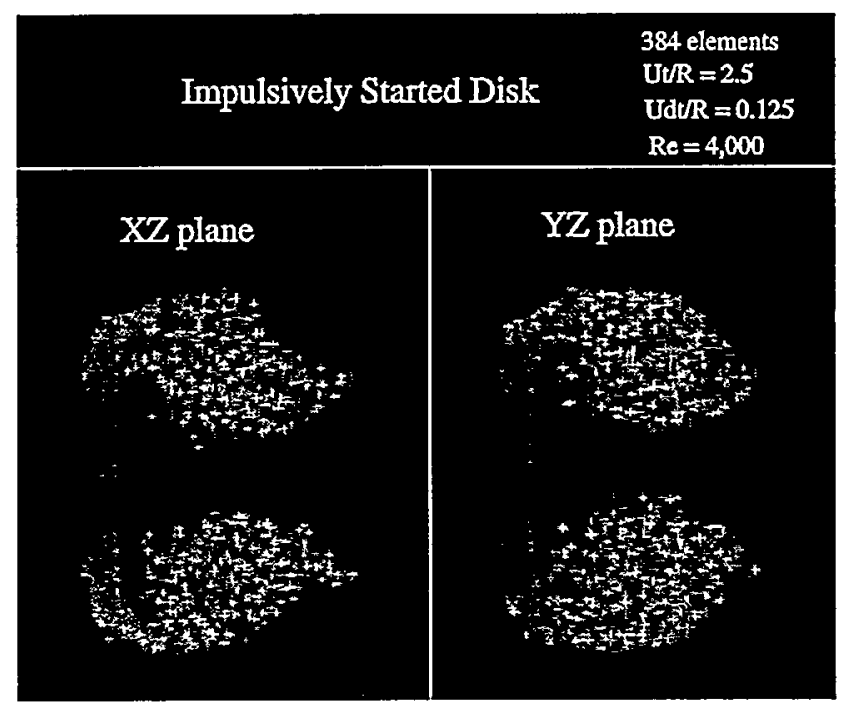

Figure 2. Impulsively Started Disk

Noticeable 3D non-axisymmetric effects in the wake can be seen. These asymmetries are most likely due to the excitation of circumferential instabilities as a result of the non-axisymmetric surface mesh. Lower Reynold's number simulations indicate a suppression of these 3D non-axisymmetric effects.

\section{Ring Slot Parachute}

We are also in the process of comparing VIPAR simulation results with experimental results from the four-ribbon "MAVEN" geometry depicted in Figure 1. These results will also be compared with those from our axisymmetric code VPARA. There are also some experimental data available for the MAVEN shape at an angle of attack. Simulation of this geometry using VIPAR will 
also require that the support struts be included in the simulation model. Wake results from a VIPAR simulation of the "MAVEN" model at an early time are shown in Figure 3. In this particular case, the body was impulsively started along the axis of symmetry ( $\mathrm{x}$ axis).

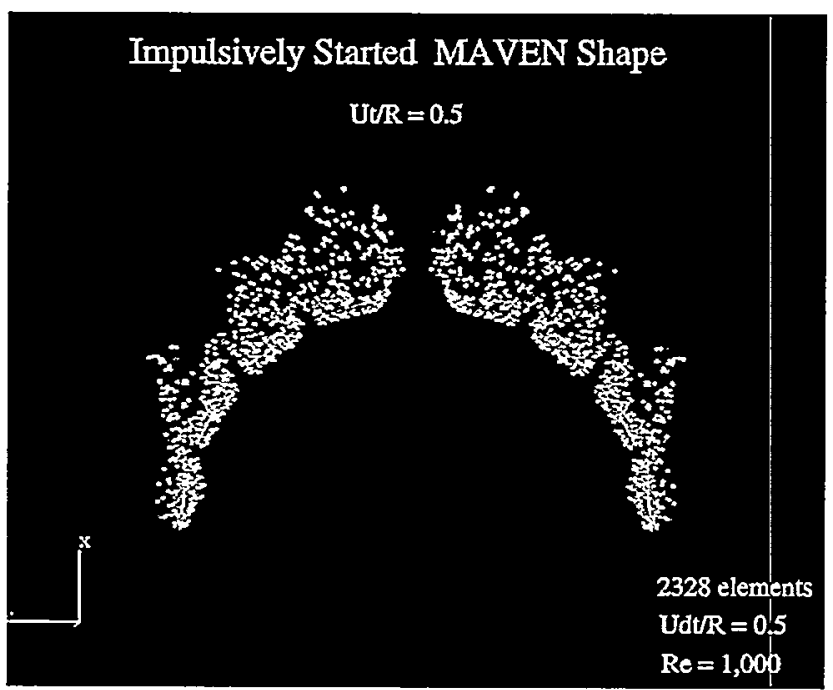

Figure 3. VIPAR Simulation of Impulsively Started "MAVEN" Model

\section{REFERENCES}

1 Peterson, C. W., Strickland, J. H., Wolfe, W. P., Sundberg, W. D., and McBride, D. D., "Development of a massively parallel parachute performance prediction code," AIAA Paper 97-1504 (A97-31302), AIAA, Aerodynamic Decelerator Systems Technology Conference, 14th, San Francisco, CA, pp. 268-276, June 3-5, 1997.

2 Behr, V. L., Wolfe, W. P., Peterson, C. W., and Hailey, C. E., "An overview of the development of a Vortex Based Inflation Code for Parachute Simulation (VIPAR)," AIAA Paper 99-1726 (A99-30980), CEAS/ AIAA Aerodynamic Decelerator Systems Technology Conference, 15th, Toulouse, France, June 8-11, 1999.
${ }^{3}$ Strickland, J. H., "A Prediction Method For Unsteady Axisymmetric Flow Over Parachutes," AIAA paper 93-1217, presented at the RAeS/AIAA 12th Aerodynamic Decelerator Systems Technology Conference, London, England, May 10-13, 1993. Also, AIAA Journal of Aircraft, Vol. 31, No. 3, pp. 637-643, May-June, 1994.

4 Sundberg, W. D., "PRONTO2D/VPARA Coupling," Internal Sandia National Laboratories Memorandum to W. P. Wolfe, January, 1999.

5 Gharakhani, A., "A 3D Galerkin Boundary Element Method for Potential Flow About Thin Membranes," 38th Aerospace Sciences Meeting \& Exhibit, Reno NV, AIAA 2000-1000, January, 2000.

${ }^{6}$ Gharakhani, A., "A Regularized Galerkin Boundary Element Method (RGBEM) for Simulating Potential Flow About Zero Thickness Bodies, Sandia National Laboratory Report, SAND99-2578, October, 1999.

7 Gharakhani, A., "A Higher Order Vorticity Redistribution Method For 3-D Diffusion in Free Space," Sandia National Laboratory Report, SAND2000-xxxx, in review, 2000.

${ }^{8}$ Homicz, G. F., and Burns, S. P., "Parallel Performance of VIPAR Fast-Solver," Memo to Distribution, Sandia National Laboratories, Albuquerque, New Mexico, September 22, 1998.

9 Taylor, L. M., Edwards, H. C., and Stewart, J. R., "Functional Requirements for Sierra Version 1.0 Beta," SAND99-2587, October, 1999. Also, see Sierra Home Page at http://www.cfd.sandia.gov/sierra.html.

10 Taylor, L. M., and Flanagan, D. P., "PRONTO3D A Three-Dimensional Transient Solid Dynamics Program," Sandia National Laboratory Report, SAND871912, March, 1989.

11 Higuchi, H., Balligand, H., and Strickland, J. H., "Numerical and Experimental Investigation of the Unsteady Axisymmetric Flow Over a Disk," Journal of Fluids and Structures, Vol. 10, No. 7. pp. 705-719, October, 1996. 